\title{
Leaving a Mobile Footprint: Utilizing Data to Combat the 2017 - 2018 Influenza Season
}

\author{
Jill D. TenHaken \\ DSRIP, Harris County Public Health, Houston, Texas, United States \\ Objective
}

During this session, participants will be able to understand how Harris County Public Health utilized data to make informed decisions on how to combat the influenza season.

\section{Introduction}

The 2017 - 2018 influenza season was classified by the Centers for Disease Control and Prevention (CDC) as 'high severity' across all age groups. Furthermore, CDC noted that this was the first year to be categorized as such, with the highest peak percentage of influenza-like-illnesses (ILI), since 2009. In Harris County alone, there were 2,665 positive flu tests reported in comparison to the previous season at 1,395 positive tests. In response to the severity of this year's flu season, Harris County Public Health (HCPH) collaborated across the department to deploy five pop up influenza vaccination events utilizing our Mobile Fleets open to the general public.

HCPH epidemiologists are able to collect influenza data from multiple systems and compile it into useful reports/tools. These data include latitudinal and longitudinal data, allowing us to create highly localized maps of where influenza has had impacted communities the hardest. This granular data allowed HCPH to target 5 areas with our Mobile Fleet that had a) high levels of influenza and b) generally limited healthcare/public health infrastructure.

Our Mobile Fleet is made up of 8 different Recreational Vehicles that have been retrofitted to offer various public health services including: immunizations, medical visits, dental visits, pet adoptions, mosquito and vector control education, and a fresh food market. The Fleet allows HCPH to offer a full menu of public health services anywhere within the County. While our efforts for this abstract were focused on controlling the influenza outbreak, we leveraged the opportunity to engage with the public on multiple issues such as environmental, veterinary, mosquito control, dental health, and accessible healthy food options.

\section{Methods}

As positive flu reports mounted, our epidemiology program provided surveillance data of influenza and ILI in Harris County. Data was obtained through multiple sources including: National Electronic Disease Surveillance System (NEDSS), which includes electronic laboratory reporting; National Respiratory Enteric Virus Surveillance System (NREVSS), which includes all flu tests done in laboratories in Houston; and last, the Flu Portal, which school nurses in Harris County upload school absenteeism rates due to ILI. Once collected and compiled, our Geographic Information System (GIS) team used the data to generate spatial maps of Harris County illustrating the disproportionally high rates. Specifically, our GIS team was able to utilize ArcGIS, and cross layer them with the flu data provided from the epidemiologists. Utilizing these maps, HCPH leadership mobilized the preparedness team to lead a data driven response in five different zip codes throughout the county to hold the influenza vaccination events.

\section{Results}

The Mobile Fleet was operational on five separate dates in five separate zip codes during February and March of 2018. Overall, 477 individuals were provided the influenza vaccine. Of those 477, 304 were 18 years or older, with 173 being under 18 years of age.

\section{Conclusions}

Having timely and actionable data is an essential first step to understand and stop an outbreak of any size. However, surveillance data alone won't prevent an outbreak from spreading. That data must be married to effective public health action. Our Mobile Fleet is able to deliver precision public health services by targeting communities most affected and vulnerable to the spread of disease. As surveillance geospatial data becomes more granular so too must our public health service delivery modes become more precise and targeted. 